\title{
A Qualitative Study on Evidence Based Practice for Filipino Physiotherapists
}

\author{
Janine Margarita Dizon ${ }^{*}, 1,2$, Karen Grimmer-Somers ${ }^{1}$ and Saravana Kumar ${ }^{1}$ \\ ${ }^{1}$ International Centre for Allied Health Evidence, University of South Australia, Australia \\ ${ }^{2}$ University of Santo Tomas, Philippines
}

\begin{abstract}
We developed and implemented an evidence-based practice (EBP) training program to a group of physiotherapists in the Philippines. This qualitative study aimed to explore the perspectives of Filipino physiotherapists regarding EBP and the EBP training provided, and to identify strategies for EBP implementation and sustainability in the Philippines. We used a qualitative descriptive design using a focus group, to answer our objectives. Maximum variation sampling was utilized, transcribed data and field notes were analyzed using content analysis, and steps to ensure rigour were noted. Seven participants took part in the focus group. Overall, EBP was perceived as essential in improving the quality of care and practice. EBP was thought to be relevant though may seem difficult, as oppose to previous reports in the literature regarding uncertainties on the relevance of EBP in developing countries. The EBP training was perceived to be effective in improving the knowledge and skills of the physiotherapists and there is definitely a need to train all health professionals to adopt an EBP culture in health care. Some strategies to sustain the EBP training program were collaboration amongst health professional organisations and integration of EBP in the undergraduate curriculum. EBP is considered to be a new concept in the Philippines, but once 'on the table' was considered to be very relevant and important in improving the quality of health care. To fully adopt a culture of EBP, nationwide EBP training in the Philippines is necessary.
\end{abstract}

Keywords: Philippines, developing country, physiotherapists, EBP, training, qualitative study.

\section{BACKGROUND}

Evidence-based practice (EBP) has been an integral component of health practices and health systems within the last ten years. Recently, the main focus of EBP has been on the uptake and implementation of practice guidelines and evaluation of the processes and outcomes known as Knowledge Translation (KT) research [1]. Health policies are now being informed by evidence from research studies such as randomized controlled trials, systematic reviews and clinical guidelines [2]. The value of applying best research evidence in order to achieve best patient outcomes in health care has highlighted the need for continuously training health professionals in EBP. In most western and developed countries, widespread EBP educational trainings in both undergraduate and postgraduate health professionals have already been in place [3-6].

Whilst EBP is a global trend in improving the quality of health care, a unique situation exists in developing countries. In developing countries, there are still concerns regarding the relevance and applicability of EBP to local contexts. These manifest as lack of knowledge and capacity in EBP, availability of good quality research studies from developing countries due to poor background on research methods, the applicability of the research evidence from developed countries and the culture of health systems (such as professional hierarchy and the lack of multidisciplinary team

*Address correspondence to this author at the International Centre for Allied Health Evidence, University of South Australia, Australia; Tel: 6188302 2099; Fax: 6188302 2853; E-mail: Janine.Dizon@unisa.edu.au approach in health care) [7, 8]. Without the appropriate knowledge and capacity to perform EBP related activities and the limited evidence base from these countries and from developed country scenarios, underpinning practice with the best evidence for best patient management seems impossible. Moreover, the culture and tradition of the population from the developing countries and the health care system (the lack of a multidisciplinary team approach in the health care setting) further challenge the uptake of EBP $[7,8]$.

To address this gap, we developed, implemented and evaluated an EBP training program, named as EBP for FilPTs, for a group of health professionals (physiotherapists) in one developing country, the Philippines (Appendix 1). We have developed the program using the concepts of complex interventions $[9,10]$ and standardizing complex interventions [11] by identifying "fixed and variable components". Complex interventions are those that include several components and a phased approach is undertaken in the process of development and evaluation [10]. The fixed component includes the core or essential functions and the variable component includes strategies in different contexts [11]. In the initial phases of the development of our EBP training program, we identified the theories, strategies and barriers to consider, that will underpin the program $[12,13]$. Moreover, we had to identify and understand the nature and the context of practice of the physiotherapists in the Philippines for us to take an appropriate approach in making the training program suitable and sustainable in the Philippine context. In our previous studies, we have highlighted that physiotherapists in the Philippines require referrals from doctors who specialize in rehabilitation 
medicine (physiatrists) and these referrals often contain a detailed treatment procedures [12]. This way of practice challenges the physiotherapists' decisions regarding patient treatment and the application of the concepts of EBP in actual practice. These iterative processes of identifying and understanding the nature and context of practice resulted to a complex EBP training program consisting of the core components of EBP (minimum requirements to teach the steps to EBP) and variable components which we innovatively created specifically for the target population (an EBP checklist and an online support) [12].

Evaluation of complex interventions necessarily involves both quantitative and qualitative research approaches [10]. Our quantitative evaluation of the EBP training program was undertaken through a pilot study followed by a randomized controlled trial (RCT). We conducted the pilot study to determine its anticipated effect in terms of EBP knowledge and skills and the program's acceptability to the local physiotherapists in preparation for the larger trial. The pilot study resulted in significant changes in pre and post EBP knowledge and skills (effect size of 0.8). Participants reported that the EBP training was effective in meeting the objectives of the program and that it was appropriate in terms of strategies and resources, to be conducted to other physiotherapists in the Philippines. Only minor modifications were required in the program that was subsequently tested in an RCT [14].

Congruent with the RCT, it is apparent that a qualitative approach was required to evaluate the complexity of participants' views regarding the first documented EBP training for a group of physiotherapists from a developing country. Thus, this qualitative study was conducted with the following objectives:

1. To explore the perspectives of Filipino physiotherapists regarding EBP

2. To explore the perspectives of Filipino physiotherapists regarding the EBP training provided

3. To identify strategies for EBP implementation and sustainability in the Philippines

\section{METHODS}

\section{Ethics}

This study was approved by the Research Ethics Committee of the University of South Australia.

\section{Design, Sampling and Data Collection Methods}

We used a qualitative descriptive study using a focus group to gather information regarding EBP and the EBP training program conducted in the Philippines. Qualitative descriptive design is used when a rich, straight description of an experience or an event is required [15]. This design seeks to obtain direct and straight descriptions of an event [16]. We used a maximum variation sampling [17] to target key informants (physiotherapists who have undergone the EBP training, physiotherapists from the education and clinical background, medical doctor who specialized in rehabilitation medicine and a medical doctor in a health research department of the Philippines) in order to address the objectives of the study. We used a focus group as our data collection method. Focus groups are used when information regarding a specific topic needs to be collected from individuals who share a common factor and that interaction among the individuals can result to greater and better insights to be developed [17].

\section{Procedures}

We sent letters of invitation to the key informants for the focus group. Information regarding the study, the objectives, the participants' role as well as the venue, date and time of the focus group were included in the invitation letters. Informed consents were signed by the participants prior to the focus group interview.

The focus group was facilitated by the primary researcher (JRD) and field notes were taken by an independent person (APA). The open ended questions asked in the interview were developed by the authors of this study (JRD, KGS and SK) (Appendix 2).

\section{Data Saturation and Rigour in the Study}

Data collection ceased when data saturation was reached. This was the point when no new information arose from the data collection method [18]. In the process of data collection through data analysis, strategies to ensure rigour were undertaken. The strategies proposed by Sandelowski 1986 [16], Guba and Lincoln 1985 [19], and Hanson 2006 [20] were integrated and used. Table 1 describes the components of rigour in qualitative research [20] and what we have specifically undertaken in this study.

\section{Data Analysis}

The interview was audio taped and transcribed for analysis. The recommended approach to analyze data for qualitative descriptive studies is content analysis [15-17]. It is defined as the categorization of verbal data and an iterative process of coding and classifying data to develop categories $[21,22]$. Transcripts from the focus group and field notes were analyzed and coded into categories and sub-categories, using content analysis. A 10-step procedure to analyze data as presented by Hancock (2002) was utilized and undertaken to ensure reliability and validity of the findings [22]. These were:

1. Read through the manuscript and noting down significant information

2. Make a list of the types of information obtained

3. Categorize items in the list

4. Sort out major and minor categories

5. Compare and contrast the categories

6. Move on to the next transcript and repeat the process from stages 1 - 5. New categories may arise but a point will be reached when no more categories can be identified

7. Collect all extracts from the interviews and place them in specific categories

8. Ensure that all items of the data are in the correct category

9. Check if the categories can fit together to form the theme 
Table 1. Methods Used to Ensure Rigour in the Study

\begin{tabular}{|c|l|}
\hline $\begin{array}{c}\text { Components of Qualitative Research } \\
\text { to Ensure Rigour [16, 19-20] }\end{array}$ & Methods Utilized in the Study \\
\hline \hline \multirow{2}{*}{ Truth value or Credibility } & $\begin{array}{l}\text { Truth value was achieved by two ways: } \\
\text { 1. Member checking (during and after the focus group interview) was done by the facilitator by summarising the } \\
\text { informants' answers to the questions to the informants, and obtaining a confirmation from them to ensure clarity } \\
\text { of responses and information provided. } \\
\text { 2. Data triangulation was done by corroborating the transcribed interview data and the field notes to identify the } \\
\text { categories and sub-categories. }\end{array}$ \\
\hline Applicability or transferability & $\begin{array}{l}\text { Applicability was ensured by utilising maximum variation sampling (we had a mixture of physiotherapists and } \\
\text { doctors), a specific type of purposive sampling, to gather rich data from key informants who can truly represent } \\
\text { the sample. }\end{array}$ \\
\hline Dependability or consistency & $\begin{array}{l}\text { Dependability was achieved by ensuring transparency of the methods, constant comparison of the categories and } \\
\text { sub-categories by the two reviewers and a third person validating the results. }\end{array}$ \\
\hline Neutrality or confirmability & $\begin{array}{l}\text { By doing a maximum variation sampling, member checking, data triangulation and constant comparison and } \\
\text { validation of categories and sub-categories formed, bias is minimised and neutrality is achieved. }\end{array}$ \\
\hline
\end{tabular}

10. Check the original copies of transcript for anything that might have been missed.

\section{RESULTS}

The focus group consisted of five physiotherapists (two completed post graduate studies) who have undertaken the EBP training, a medical doctor who specialized in rehabilitation medicine (with post graduate degree in health research) and a medical doctor who specialized in internal medicine and clinical research. Two of the five physiotherapists in the group represent the Philippine Physiotherapy Association (PPTA), the professional organization of physiotherapy in the country. The medical doctor with a specialization in rehabilitation medicine also represents the Philippine Academy of Rehabilitation Medicine (PARM), the national society of doctors who specialized in rehabilitation medicine. The medical doctor with a specialization in internal medicine and clinical research represents the Philippine Council for Health Research and Development (PCHRD). The members of the group have been in practice (in the clinical, education and research areas) from eight to twenty years.

There were five main categories that emerged from the focus group regarding the EBP training (See Fig. 1):

\section{Perspectives in EBP}

2. Feedback on the EBP training

3. Perspectives in applying the concepts of EBP in actual practice

4. Difficulties encountered/ barriers to applying EBP in actual practice

5. Perspectives about providing ongoing EBP training and strategies for sustainability of EBP in the Philippines

\section{Perspectives in EBP}

The perspectives of the members of the group regarding EBP can be summarized in three main sub categories which are: essential in improving quality of care, an innovation and perceived barriers. These perspectives reflect that whilst EBP is a new concept to the participants, they have valued what EBP can contribute to the quality of local health care. These perspectives also reflect that whilst they have identified potential local barriers, EBP is still relevant to the Philippines.

\section{Essential in Improving Quality of Care}

EBP has been viewed as important in improving the quality of health care and setting standards in practice. The main goal of every health professional is to achieve best patient outcomes and EBP is essential in achieving this.

"EBP is important because its ultimate benefit to society is improvement in the quality of care given to patients. Improvement of quality of patient care is through improving the standard of practice in the health profession."

"EBP is important to improve practice and patient care. I believe that EBP should be an integral part of practice and therefore, we all need to be trained in EBP."

\section{$E B P$ is an Innovation}

EBP is viewed by members of the focus group as something new and is a good and engaging update in practice.

"EBP is something new in practice as it exposes individuals to new knowledge and thinking."

"EBP is good and engaging. It facilitates health professionals to think in a different way and answers a lot of questions."

\section{Perceived Barriers}

Participants believe that whilst EBP is important in improving the quality of health care, it seems difficult and there are issues to consider if it really can be done and applied in the Philippines.

\section{"EBP may be difficult but I think it is possible in the Philippines. Sometimes, very few literature are relevant to us."}

"EBP is important but we need to review research methods" 


\section{EBP for FilPTs \\ (considered as complex interventions with fixed and variable components)}

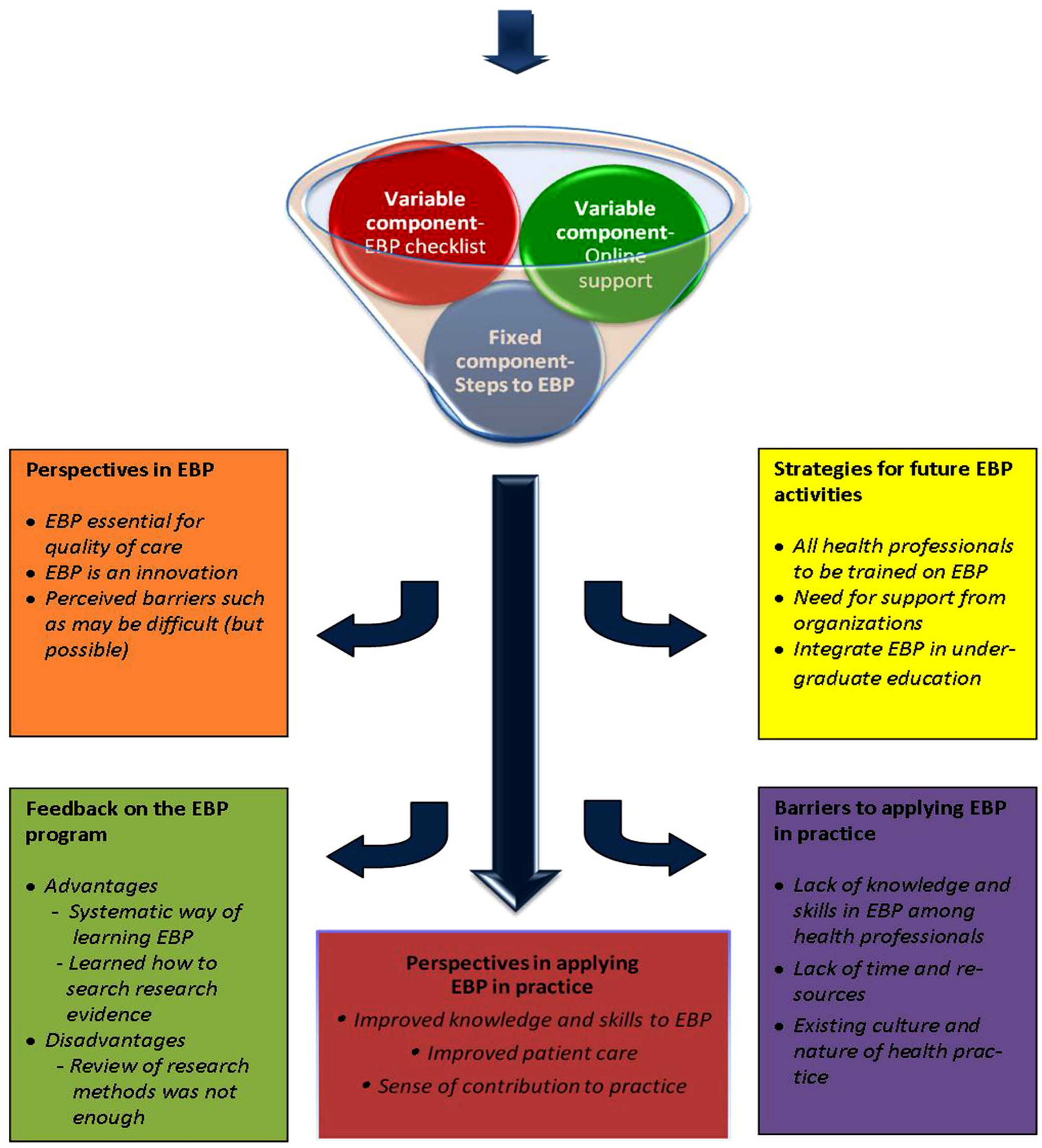

Fig. (1). Categories and sub-categories arising from the focus group regarding the EBP for FiLPTs.

"EBP raises the question, can we really do it? There are inter-professional issues that need to be considered."

\section{Feedback on the EBP Training Program}

The feedback on the EBP training program were further sub-categorised into: advantages, disadvantages and recommendations for improvement. The participants' perspectives regarding the EBP training are essential in order to build on its strengths and modify the contents, strategies and methods of conducting the program, as needed, for a more relevant and context specific program for the Filipino physiotherapists.

\section{Advantages of the EBP Training Program (What Worked for the Participants)}

Participants who have undergone the EBP training believed that they have improved knowledge (EBP steps and 
concepts) and skills (searching and appraising) in EBP after the training. They have acknowledged that the content and format of the training were effective in improving these domains of EBP. They have appreciated the access to the electronic databases provided during the training which enhanced the teaching.

"The content and format of the lectures in the training were effective. The training provided a formal and systematic way of learning EBP"

"The training taught me to analyze research articles and I have learned to access the evidence using different electronic resources provided during the training "

"I have learned to maximize the use of Google Scholar with the training"

\section{Disadvantages}

"The training did not cover the critical appraisal of other research designs".

"The training was helpful but we need more review on research methods."

\section{Recommendations for Future Training (Content and Structure)}

Whilst the participants acknowledged that the lectures and practical sessions in the EBP training were effective and they have learned much in only one day, they have also identified ways to further improve the EBP training program and these focused on the content and structure of the program.

"What was taught in the training and the way the lectures and practical sessions were presented were the best for me."

"I think critical appraisal of all research studies should be taught in the training”.

"There should be a review of basic research methods. Those with good research background can follow quickly, while others may not. Although you have explained the lectures well, in reality, other people may need more basic review of research."

\section{Perspectives Regarding the EBP Training when Applied in Actual Practice}

Participants viewed the EBP training as useful in improving their knowledge and skills, improving the quality of care and a sense of contribution to the practice. The participants' perspectives regarding applying EBP in practice reflect the practical implications when using the knowledge and skills learned in the training in real work scenario. This information now reflects the true sense of relevance and applicability of the EBP training program and how it facilitates good clinical decision making.

\section{Improved EBP Knowledge and Skills}

"I was able to search for best and up to date evidence on what to do in practice. Before I only use textbooks as reference."
"I learned about the importance and use of systematic reviews and present the most up to date research evidence to negotiate with medical doctors whom I work with."

"From research, I have learned how to choose the best treatment for my patient. If the treatment does not work for a particular patient, then I choose the next best treatment."

\section{Improving Quality of Care}

"Applying the principles EBP in practice helped improved my clinical decision making skills and helped me provide better care for my patient. It helped me identify which intervention to use based on what the evidence says and based on what the patient wants."

"To maximize the provision of quality health care to patients, all health professionals should undergo the EBP training; most physiotherapists can't make decisions without a rehabilitation doctor's consent."

\section{Sense of Contributing to the Practice}

"I appreciated the fact that there is a sound basis for what I do in practice after learning the concepts of EBP. I was able to improve my clinical education training for my interns (PT students on practical placement) by sharing what I have learned and the updated information from research, which I think is good as I have updated them also.

"It felt good to be able to identify and present updated findings to doctors, other health professionals and students and being acknowledged for it.

"I was able to share what I have learned with my colleagues."

\section{Difficulties Encountered/ Barriers to Applying EBP in Practice}

Barriers identified were related to lack of knowledge and skills (in EBP and regarding treatment interventions), time, resources and the culture and nature of health system. As expected with any change in the usual practice, barriers can be encountered. Identifying barriers to applying EBP helps to identify potentially effective strategies to minimise the barriers for a more effective program.

\section{Lack of Knowledge and Skills in EBP}

"It was difficult to engage in discussions with some health professionals who were not familiar with EBP."

\section{Lack of Time}

"We had less time to search and appraise the evidence when we went back to work."

\section{Lack of Resources}

"I had difficulty accessing a computer with internet connection at work." 
"I had difficulty accessing the evidence outside the training especially when I experience slow internet connection."

\section{Culture and Nature of Health Systems}

"There is a need to educate all health professionals in EBP so that we can all engage in discussions regarding the best management for the patient. If some health professionals are not open to recommendations regarding treatment from us who have learned about $E B P$, then we cannot deliver treatment that is based on what really works. Each health professional has a role in the health system and therefore, a multidisciplinary team approach is needed."

Perspectives About Providing Ongoing EBP Training and Strategies for Sustaining the EBP Program in the Philippines

Participants agreed that there is a need for EBP training to up skill the local physiotherapists and all other health professionals involved in managing the patient, such as rehabilitation doctors and nurses and even undergraduate students. As the EBP training has been reported to be contextually appropriate for the Filipino physiotherapists, long term implementation plans and strategies have been identified. With the lack of time and limited resources, a need for support to sustain EBP related activities should be in place.

"There really is a need for a widespread EBP training in the country and this EBP training provided to us should continue."

"All health professionals should undergo training in EBP so that recommendations based on evidence can be applied in practice."

"For EBP to be fully adopted in the country, it should be integrated in the undergraduate curriculum."

"Professional organizations should collaborate and get involved in programs to promote EBP.”

"Identify institutions that can fund and support trainings in terms of resources."

\section{DISCUSSION}

This is the first qualitative study to our knowledge, which evaluates an EBP training delivered to a group of physiotherapists from a developing country (in this case, the Philippines). This study highlighted important findings regarding:

1. The relevance and importance of EBP in health care in the Philippines,

2. Feedback regarding the EBP training program and applying EBP knowledge and skills learned in practice and,

3. Barriers and strategies for implementing and sustaining EBP activities in the Philippines.
Perceptions on EBP and Issues Regarding the Relevance and Importance of EBP in Health Care in the Philippines

Overall, EBP was considered by participants as a new concept and an innovation in practice. EBP is important and valuable in improving the quality of health care and setting standards in practice whether in developed or in developing countries. The ultimate aims of using evidence to guide practice are to improve health care and achieve best patient outcomes [1-2]. Whilst there are issues in developing countries regarding EBP, the availability and relevance of the current evidence, the realism of EBP to developing country scenarios and settings, and the limited time, access and resources, there are ways to address such issues to move forward to adopt a culture of EBP. Given that there is limited published research reflecting developing country scenarios, the focus of EBP in developing countries should be on localizing the decision from the global evidence [23]. It is essential that health professionals learn how use their clinical expertise to localise the decision by adapting the available good quality evidence to the needs of the patients, the local setting and the available resources. However, we believe that prior to learning how to localise the decision, health professionals need to learn the essential knowledge regarding the concepts of EBP, thus the importance of providing an EBP training program (such as the EBP for FilPTs), that covers this component.

In addition to the concepts of EBP as a core component of EBP training programs, strategies to apply the "adapted and localized decision" to patients should be included in the program. Strategies that are aimed at influencing behaviors need to be underpinned by the characteristics and the needs of the target population, and their local setting of practice $[10,24]$. When these important characteristics of the population and the health care setting have not been considered, they can be barriers to the application of the evidence. In our program, the strategies we developed to address these concerns were (1) the use of an EBP checklist and (2) an online support that is freely accessible [12, 14]. The EBP checklist was our strategy to enable physiotherapists to make recommendations and negotiate with the doctors (physiatrists) regarding treatment interventions with evidence for effectiveness, if there is a need to do so. The online support which is a link where physiotherapists can log their clinical question and PICO (Ppopulation, I-intervention, $\mathrm{C}$-comparison and $\mathrm{O}$-outcome) to seek assistance in finding the evidence was our strategy to address the lack of time and access. These strategies were valued by the participants who undertook the training. However they also had difficulty in recommending some treatment interventions to other health professionals (doctors and nurses) who were not familiar with EBP. This reverts back to one of the main barriers which is the lack of fundamental knowledge and existing misconceptions regarding EBP, thus highlighting again, the need to provide training and educate other health professionals in EBP.

Feedback Regarding the EBP Training Program and Applying EBP Knowledge and Skills Learned in Practice

The content and format of the EBP training program were considered to be effective in improving knowledge, skills and attitudes regarding EBP. Resources provided were 
appreciated by the participants (venue, availability of computers to use and access to databases). Searching and appraisal skills and use of research information (such as systematic reviews rather than textbooks only) and decision making skills were perceived to be improved as a result of attendance in the EBP program. Providing the essential resources for training has a good impact on the learning of participants as they can practice the skills taught to them. Building on these skills that were developed (searching, information use and decision making) is important in enhancing a culture of EBP in the system [25].

A major recommendation of the participants of the focus group was a need for a review on research methods and statistics. The participants felt this was needed in order for them to understand the statistical tests and significance of the findings. One of the issues of developing countries regarding the use of EBP is poor background on research methods [8]. Whilst we have considered such in the development of the EBP training program and included a lecture on review of research designs, this may have not been enough and we are considering on providing a short but focused preliminary course on health research methods and statistics (to those who think it is needed) prior to the EBP training.

The EBP training program resulted to improved perspectives of the participants regarding a multidisciplinary team approach when applied in practice. The participants valued the roles each health care professional played in the health system when they accessed and read more research articles. Multidisciplinary team approach in the health care results to better patient management and outcomes [26]. In this approach, it is ensured that all aspects of a patient's condition are discussed and managed by the health professional who has the specific knowledge and skills in relation to the patient's needs.

An interesting finding in this study was the sense of contribution to the practice felt by some physiotherapists who had the training and applied the concepts of EBP in discussions amongst interns (undergraduate physiotherapy students who are in the last year of their degree and undertaking clinical placement). The improved capacity of the physiotherapists in EBP was translated to the interns who are the next generation of clinicians. Educating the interns on EBP influences their way of thinking and improving their clinical decision making skills in the clinical setting (as opposed to classroom teaching). Empowering clinicians in this manner enhances mentorship which is one of the facilitators of clinical education teaching $[27,28]$. This then can contribute to enhancing a culture of EBP in the health system.

\section{Barriers and Strategies for Implementing and Sustaining EBP Activities in the Philippines}

The barriers identified by the members of the focus group reflect similar barriers reported in the literature such as lack of knowledge and skills to EBP, lack of time and lack of resources [29-31]. It was strongly acknowledged that there is a need to extend the training to other health professionals in the Philippines to address the lack of knowledge and skills. Moreover, there is a need to include EBP in the undergraduate curriculum to teach EBP in the earlier stages of professional training. The training program raised awareness in EBP and it is very important that efforts should be directed to addressing the barriers and sustaining the program to adpot a culture of EBP in the country.

Some useful strategies to implement EBP in the Philippines were involving professional organizations in the activity and endorsing the EBP training, collaborative efforts from health professional organizations in planning activities related to EBP and support from funding institutions. These strategies are all in accordance to what the literature suggests in implementing EBP in the system [32]

\section{Implications for Practice}

- $\quad$ The EBP training program, would some refinements in its content and structure, can be delivered to other health professionals to raise awareness and improve capacity in EBP. A nationwide training in EBP in the Philippines is fundamental to bridge the knowledge gap among health professionals.

- With the increased knowledge and skills in EBP among health professionals, strategies to achieve a multi disciplinary team approach in making decisions regarding health care can be promising.

- $\quad$ The EBP training delivered to physiotherapists in the Philippines can be a model for other developing countries faced with similar concerns.

- We have focused on exploring the perspectives of physiotherapists from a developing country regarding EBP but the practical implications of localizing the decision from the global evidence considering the characteristics of the population and the health care setting is be a global undertaking and can be useful in developed countries as well.

\section{Implications for Research}

- $\quad$ Future research needs to focus on development and evaluation of culturally relevant EBP training programs and the impact on real practice (patient and workplace) scenario

- Future research also needs to focus on the best methods to teach EBP amongst undergraduate health professionals' training specific to the culture of teaching and learning among developing countries.

\section{CONCLUSION}

EBP is considered to be a new concept in the Philippines, but once 'on the table' was considered to be very relevant and important in improving the quality of health care. The EBP training program delivered to physiotherapists in our research was a valued undertaking in promoting EBP in the Philippines and should be sustained. Strategies to sustain the training and extend it to other health professionals have been identified. EBP needs to be integrated in the education and health systems to eventually adopt a culture of EBP in the country.

\section{ACKNOWLEDGEMENT}

Declared none.

\section{CONFLICT OF INTEREST}

The authors declare no competing interest 


\section{APPENDICES}

\section{Appendix 1}

\section{Outline of the Training Program}

\section{EBP Training for Filipino Physiotherapists}

\section{Program Objectives}

The tailored Evidence Based Practice (EBP) program for Filipino physiotherapists aims to teach the concepts of EBP in a manner fitting the practice of physiotherapy in the Philippines. The EBP program also aims to develop evidence seeking pattern and behaviour among the physiotherapists and therefore update their existing knowledge and enhance the use of the best evidence to guide practice in the long term process.

\section{Specific:}

1. To teach the concepts of EBP

2. To teach clinical question formulation using the PICO format (based on a scenario where clinician is faced with a new or unique case)

3. To teach participants how to search for the best evidence to answer the clinical question (including hierarchy of evidence and sources of the best available evidence)

4. To teach critical appraisal skills to assess the quality of the evidence found

5. To apply the knowledge and skills learned to draft an answer the question identified

6. To assess the process from question formulation to applying the skills learned

\section{Strategies}

Lecture and demonstration

Actual practice of skills

Problem solving

Post training support

\section{Assessment}

To objectively measures changes in EBP knowledge, skills and attitudes, the Adapted Fresno Test (McCluskey and Bishop 2009) and the Questions on EBP Attitudes (Stevenson, Lewis and Hay 2003) will be administered before and after the EBP program.

Activity diaries would be provided for participants to log activities (within 3 months post training) related to searching for answers to new or unique clinical cases.

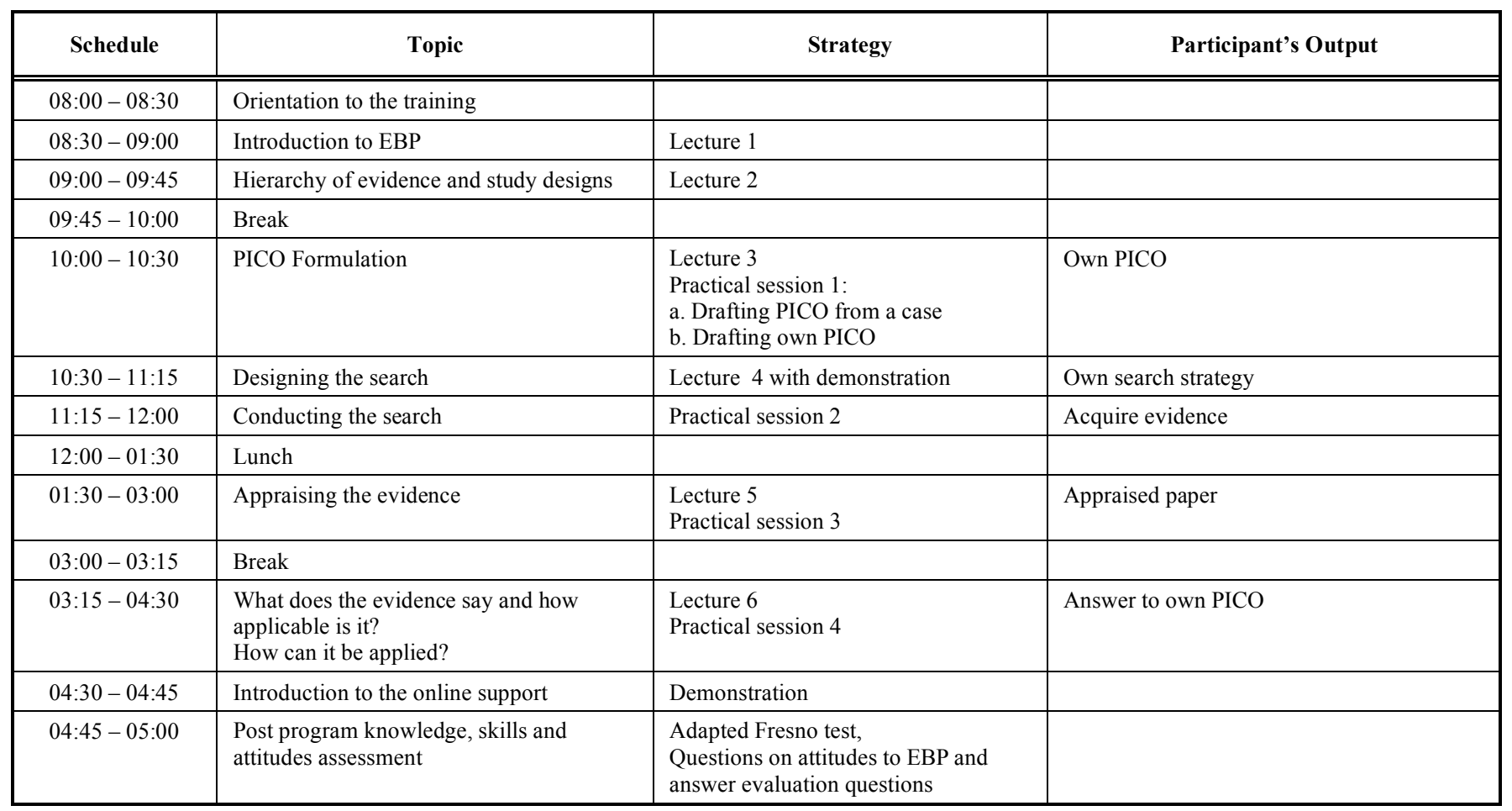




\section{Appendix 2}

The open ended questions asked in the interview:

1. What are your perspectives of EBP in Philippines and the EBP training conducted?

2. For those who have undergone the training, what do you think worked for you and what didn't? (advantages and disadvantages)

3. If we were to run this training again, what, if any, recommendations will you have for any improvement? (in terms of format, duration and applicability to the Filipino physiotherapists)?

4. In what ways was the learning from the training useful and applicable to actual practice?

5. What were the difficulties encountered or barriers to applying the learning from the training to actual practice?

6. Now that you have been part of EBP training and have heard feedback about it, what are your perspectives about providing ongoing EBP training for Filipino PTs?

7. What strategies will facilitate the sustainability of the EBP training and eventually evidence uptake and implementation in the Philippine setting?

Note that the questions in bold and italics were answered by ALL members of the group while those in regular font were answered only by those who took part of the training.

\section{REFERENCES}

[1] Curan JA, Grimshaw JM, Hayden JA, Campbell B. Knowledge translation research: the science of moving research into policy and practice. J Contin Educ Health Prof 2011; 20(4): 238-46.

[2] Overholt EF, Melnyk BM, Schultz A. Transforming health care from the inside out: advancing evidence-based practice in the $21^{\text {st }}$ century. J Prof Nurs 2005; 21(6): 335-44.

[3] Burns HK. Building a foundation for an evidence-based approach to practice: teaching basic concepts to undergraduate freshman students. J Prof Nurs 2005; 21(6): 351.

[4] Martin SD. Teaching evidence-based practice to undergraduate nursing students: overcoming obstacles. J Col Teach Learn 2007; 4(1): 103.

[5] Glasziou P, Burls A, Gilbert R. Evidence based medicine and the medical curriculum. The search engine is now as essential as the stethoscope. BMJ 2008; 337: 704-5.

[6] Chipchase LS, William MT, Robertson VJ. Factors affecting curriculum content and the integration of evidence-based practice in entry-level physiotherapy programs. J Allied Health 2007; 36(1): 17-37.

[7] Chinnock P, Siegfried N, Clarke M. Is evidence-based medicine relevant to the developing world? PLoS Med 2005; 2 (5): e107

[8] Agarwal R, Kalita J, Misra UK. Barriers to evidence-based medicine practice in South Asia and possible solutions. Neurol Asia 2008; 13: 87-94.

[9] Dizon JM, Grimmer SK. Complex interventions required to comprehensively educate allied health practitioners on evidencebased practice. Adv Med Educ Pract 2011; 2: 105-8.
[10] Campbell M, Fitzpatrick R, Haines A, et al. Framework for design and evaluation of complex interventions to improve health. BMJ 2000; 6: 321-94.

[11] Hawe P, Shiell A, Riley T. Complex interventions: how "out of control" can a randomized controlled trial be? BMJ 2004; 328: 1561-3.

[12] Dizon JM, Grimmer SK, Kumar S. Effectiveness of the tailored EBP training program for Filipino physiotherapists: A randomised controlled trial, study protocol. BMC Med Educ 2011; 11: 14.

[13] Dizon JM, Grimmer SK, Kumar S. The physical therapy profile questionnaire (PTPQ): development, validation and pilot testing. BMC Res Notes 2011; 4 (362) doi: 10.1186/1756-0500-4-362.

[14] Dizon JM, Grimmer SK, Kumar S. A pilot study of the evidence based practice training program for Filipino physiotherapists: emerging evidence on outcomes and acceptability. Int $\mathrm{J}$ Allied Health Sci Prac 2012; 10 (2): 1-12

[15] Neergaard MA, Olesen F, Andersen RS, Sondergaard J. Qualitative description - the poor cousin of health research? BMC Med Res Methodol 2009; 9: 52.

[16] Sandelowski M. Focus on research methods whatever happened to qualitative description? Res Nurs Health 2000; 23: 334-40.

[17] Patton M. Qualitative Research and evaluation methods. Thousand Oaks: Sage 2002.

[18] Rice P, Ezzy D. Qualitative research methods: a health focus. Oxford: Oxford University Press 1999.

[19] Guba E, Lincoln Y. Effective evaluation. San Francisco: JosseyBass 1981

[20] Hanson E. Successful qualitative health research. Crows Nest NSW: Allen \& Unwin 2006.

[21] Mays N, Pope C. Qualitative research: observational methods in health care settings. BMJ 1995; 311: 182-4.

[22] Hancock B. Trent focus for research and development in primary health care: An introduction to qualitative research. UK: Trent Focus 2002.

[23] Eisenberg JM. Globalize the evidence, localize the decision: evidence-based medicine and international diversity. Health Affairs 2002; 21(3): 166-8.

[24] Michie S, Van Stralen MM, West R. The behaviour change wheel: a new method for characterising and designing behaviour change interventions. Implement Sci 2011; 6: 42.

[25] Dawes M, Summerskill W, Glasziou P, et al. Sicily statement on evidence-based practice. BMC Med Educ 2005; 5(1): 1-7.

[26] Lemieux CL, McGuire W. What do we know about health care team effectiveness? a review of the literature. Med Care Res Rev 2006; 63(3): 263-300.

[27] Lekkas P, Larsen T, Kumar S, et al. No model of clinical education for physiotherapy students is superior to another: a systematic review. Aust J Physiother 2007; 53: 19-28.

[28] Lambert V, Glacken M. Clinical education facilitators: a literature review. J Clin Nurs 2005; 14(6): 664-73.

[29] Maher C, Sherrington C, Elkins M, Herbert R, Moseley A, Grimmer K. Challenges for evidence-based physical therapy: accessing and interpreting high-quality evidence on therapy. Phys Ther 2004; 84(7): 644-54.

[30] Grimmer K, Bialocerkowski A, Kumar S, Milanese S. Implementing evidence in clinical practice: the 'therapies' dilemma. Physiotherapy 2004; 90: 189-94.

[31] Grimmer SK, Lekkas P, Nyland L, Young A, Kumar S. Perspectives on research evidence and clinical practice: a survey of Australian physiotherapists. Physiother Res Int 2007; 12(3): 14761.

[32] Grol R, Grimshaw J. From best evidence to best practice: effective implementation of change in patients' care. Lancet 2003; 362: 1225-30. 